\title{
Research on Shuttlecock Activity for Special Big Break in Regular College
}

\author{
Bin Cao \\ Shandong Female College \\ Jinan, China
}

\begin{abstract}
Currently, since college students have difficulties in being interested in exercising, it is required to focus on mentality and physiology of college students; what's more, Notification of Implementing Sunshine Sports for Hundreds of Millions Students in China was issued by Ministry of Education, State General Administration of Sports and the Communist Youth League. In this case, we intend to have a continuous research on How to Build up the Body of College Students, especially Female Students. So we had a questionnaire about the interest in shuttlecock and participation situation in terms of 500 college students. The questionnaire is able to help us a lot with the implementation of shuttlecock. And by the questionnaire, we hope that how to improve the college students' sense of sports should be figured out, as well as how to build up the body and how to truly take part into sports.
\end{abstract}

Keywords-College students; sports during break; shuttlecock; sense activity

\section{Introduction}

Kicking Shuttlecock was originated from civilians, with profound cultural connotation. There are two different kinds of shuttlecocks which are synchronized shuttlecock and shuttlecock. It is an emerging sport which had been developed rapidly in recent years, which is characterized by entertainment, moveability, mass, fitness, ubiquity and edutainment. In addition, as shuttlecock is interesting, healthy and enjoyable for being watched, there are more and more people are in love with it. The shuttlecock being used now is updated from the traditional one, being combined with elements of competitive sports, so it is not only full of fitness, interest and enjoyability, but also antagonism. At present, Chinese shuttlecock is able to become an international sport.

\section{DOMESTIC RESEARCH STATUS}

Research on Implementation of Sunshine Big Break for School Physical. Regarding the background of Sunshine Sports, the research is mainly about the necessity of big break as well as the organization of implementation.

Feasibility research on introducing shuttlecock to colleges. Currently, the main researches are: know about the shuttlecock faculty by questionnaire and interview, as well as students' participation of shuttlecock; a further research on the feasibility of introducing shuttlecock to colleges; also, feasibility research on theories about characteristics, functions of shuttlecock as well as implementation of big break.

Research on Technology of Shuttlecock. The research is mainly about the implementation of shuttlecock in terms of the development of shuttlecock, technique and tactics, training method and principals etc.

\section{RESEARCH SIGNIFICANCE OF SHUTTLECOCK}

\section{A. Shuttlecock is a Traditional Sport}

Shuttlecock is one of the traditional ethnic sports. Ethnic sport is full of cultural deposits; it is a kind of sports cultural wealth; it is an important part of native sports. Therefore, the value of shuttlecock should be supported by physical education. School is a critical base for transferring physical culture, by which the excellent folk sports will be combined with school physical education. The usability and infectivity of school physical education will be badly improved and enhanced, which will become a part of modern sports culture education. What's more important, it means that the folk sports are being involved into modern school sports education. The characteristics of shuttlecock, like universality, interest, convenience, fitness and entertainment, comply with the mental and physical development of college students, as well as their hobbies; additionally, the characteristics comply with the requirements of sports education reform requested by quality-oriented education; the characteristics comply with the guiding ideology of development first. In a word, the promotion and avocations of shuttlecock in colleges will be beneficial of the development characterized by national features and the extension of Chinese culture.

\section{B. To meet the necessity of "Sunshine Big Break"}

In December, 2006, there was Notification of Implementing Sunshine Sports for Hundreds of Millions Students in China (short for Sunshine Sports) issued by Ministry of Education, State General Administration of Sports and the Communist Youth League, requiring that all schools should consider the sunshine sports as a breakthrough and major work for wholly promoting quality-oriented education. Sunshine Sports should be carried out in colleges, high schools and primary schools within China, trying to achieve that each people know and 
understand the slogan "Health first", "One hour for sports every day for fifty years of healthy work. will be realized and a happy life will be followed". Try to achieve that more than $85 \%$ schools are able to carry out Fitness and Health Standard of Students; more than $85 \%$ students are able to do sports for one hour one day, being qualified or more of Fitness and Health Standard of Students and knowing about at least 2 kinds of sports; so at last, an atmosphere that ordinary people are able to take part into mass sports.

\section{Necessity of College Students' Physical and Psychological Health}

The definition of health confirmed by World Health Organization is not only one is healthy physically, but also health psychologically; and one is able to adapt to the society and be healthy in morality. Doing sports is the best way to be healthier. Since shuttlecock is an emerging sports which is simple to be learned and diversified in exercising (exercised by one people or two people or even more), so by implementing special shuttlecock activity during big break in Shandong Female College, the campus atmosphere will be activated, health conditions will be improved and sense of team-work spirit among students will be enhanced. According to relevant research, if one take part in shuttlecock frequently, the flexibility of muscle and ligament of lower limbs will be strengthened, and the arthrosis will be more smart; what's more, the heart and pulmonary system will be exercised which will improve one's healthy status. When several students are in a group to play shuttlecock, they will feel highly relax and try to kick the shuttlecock with different body parts. When play shuttlecock, fun and pleasure will be created by receiving and kicking the shuttlecock. So the students will be in a good mood which will hugely help them with their study.

\section{Physiological Feature of Female Movement}

As for women, the bones are small which is not so capable of deflection resistance and crumpling resistance. However, the joint ligament is superior in flexibility and smartness. Hence, intensive sports is not suitable for women to take part in for a long time; since shuttlecock is not an intensive sport and there are kinds of diversified exercising methods, requiring good flexibility and smartness which are the advantages of women. In a word, feasibility of implementing shuttlecock during big break is very considerable. At present, most of researches are being focused on bringing in shuttlecock into colleges, while implementing shuttlecock during big break is seldom. Based on my serious survey of large amounts of documents, it was founded that it is very important and practical to have a research on the implementing shuttlecock during big break in regular female colleges.

\section{E. Formation of Lifelong Sports}

College time is considered as a golden period for students to develop sports habit and master sports skill. It is also a key period for students to develop sense of sports. During college time, how to motivate students' interest and how to master skills of one or two sports for students is really important and critical. As a team-work sports after class, special big break of shuttlecock is able to make the students relax after class, to develop students' sports skill in a good atmosphere. In this case, the students' sense of sports will be strengthened gradually.

\section{F. Features of Shuttlecock}

Shuttlecock is a kind of sports which is full of interest, enjoyability, fitness and antagonism. Since shuttlecock was originated from civilians, so people are familiar with it that most of the people are able to play. Shuttlecock is easy to learn and cheap to make that basically each people is able to afford. Even, some are able to make a shuttlecock by themselves. As for colleges, when there is spare time after class, the students can be organized to make shuttlecocks. Notwithstanding, with regard to aforementioned, female colleges own advantages. Therefore, it is really necessary to do the research on implementing shuttlecock in female colleges.

\section{G. Necessity of Implementing Shuttlecock in Colleges}

Sports implemented in colleges required that the sports should have the function of fitness, team-work and entertainment, as well as developing the determined spirit. Shuttlecock fully complies with these kinds of requirements. That is to say, shuttlecock not only enriches the sports activity in colleges, but also meet the diversify requirements of college students. Currently, balls and athletics are the main sports in most schools, which is not able to satisfy the requirements of students. So if shuttlecock is promoting in colleges, sports activity will be more diversify and flexible. In this way, the requirements of sports reform will be satisfied and the sports culture will be promoted; what's more, the combination of school sports and national sports will be sped up.

\section{H. Value of Interpersonal Communication Exercising}

As for most college students, they are not capable enough to adapt to the society. It is a key problem that interpersonal communication is hugely lack. As for shuttlecock, 2 people are required to be involved which will make the participated students try to have interpersonal communication with others. So when play shuttlecock, it not only enforces the communication among students, but also provides opportunities of mutual-study and experience-learning. Additionally, the students' motivation in learning shuttlecock is to be enhanced and the necessity in communicating with others is to be strengthened. So gradually, the social ability will be improved which will be helpful in terms of moving forward to the society.

\section{Requirements of Shuttlecock Instructors in College}

Shuttlecock instructors in colleges are the major player of shuttlecock, who needs to master the psychological change of students when they are playing shuttlecock, trying to eliminate students' bad habits and psychological barriers. In order to achieve the goal aforementioned, it is required that the teachers should improve their own qualities in terms of mastering large amounts of psychological knowledge and 
education methods of PE. In order to have an efficient instruction on shuttlecock, it is required that the instructors should continuously improve their method and ideology; have the students done psychological training and psychological control by different kinds and different methods.

\section{MAIN RESEARCH CONTENT AND BASIC IDEOLOGY}

\section{A. Example of Research}

Analyze the implementation and development of shuttlecock activity in Jinan University, as well as the process of shuttlecock entering campus. By reading relevant research and analyzing the feasibility of shuttlecock entering campus, try to analyze the mode of shuttlecock entering campus. On the basis of relevant research, the author is trying to analyze the theme, combining with the characteristics of age, gender and time of female college students.

\section{B. Emphasis and Difficulties}

On one hand, with respect to the read documents, there are few researches on "Sunshine Big Break" of regular female colleges and there is no relevant research on implementing shuttlecock in female colleges during big break. Therefore, my research will be innovative. On the other hand, it is difficult that the implementation mode of shuttlecock in high and primary schools should be involved into that of regular female colleges.

\section{Major Points and Innovation}

When reading literatures and collected documents, the author believed that the feasibility of implementing shuttlecock activity during big break in regular female college is superbly high, where there are sole points of implementation mode. In the view of innovation, the research on implementing shuttlecock activity during big break is innovative and creative because there is no similar research when collecting documents.

\section{REFERENCES}

[1] ZHANG Haiping etc. Practice Research on Sports during Big Break [J].Sports Science and Technology, 2002, 23 (3) : 33-34

[2] HOU Fulu. Primary Research on Sports during Big Break in Higher Vocational College [J]. PE World, 2012, 6:8-9

[3] LIU Lizhen. Brief Introduction of Big Break Activity [J]. Sports, 2012, 6: 111

[4] FENG Jun. Research on Implementation Strategy of Big Break School-based [J].Window of Sports Art, 2010, 5: 74 